\title{
Dopaminergic-induced dyskinesia assessment based on a single belt-worn accelerometer
}

Carlos Pérez-López a, Albert Samà a , Daniel Rodríguez-Martín a , Juan Manuel MorenoAróstegui a , Joan Cabestany a , Angels Bayes ${ }^{\mathrm{b}}$, Berta Mestre ${ }^{\mathrm{b}}$, Sheila Alcaine ${ }^{\mathrm{b}}$, Paola Quispe ${ }^{\mathrm{b}}$, Geroid Ó Laighin ${ }^{c}$, Dean Sweeney ${ }^{c}$, Leo R. Quinlan ${ }^{\mathrm{d}}$, Timothy J. Counihan ${ }^{\mathrm{e}}$, Patrick Browne $^{\mathrm{e}}$, Roberta Annicchiarico ${ }^{\mathrm{f}}$, Alberto Costa ${ }^{\mathrm{f}, \mathrm{g}}$, Hadas Lewy ${ }^{\mathrm{h}}$, Alejandro RodríguezMolinero $^{c}$.

${ }^{a}$ Centro de Estudios para la Dependencia y la vida Autónoma (CETpD), Universitat Politècnica de Catalunya (UPC), Rambla de l'Exposició, 59, 08800 Vilanova i la Geltrú, Barcelona, Spain

${ }^{\mathrm{b}}$ UParkinson, Barcelona, Passeig Bonanova 26,08022 Barcelona, Spain.

${ }^{\mathrm{c}}$ Electrical \& Electronic Engineering, School of Engineering \& Informatics National University Galway (NUIG), University Rd, Galway, Ireland

d Physiology, School of Medicine National University Galway (NUIG), University Rd, Galway, Ireland

e School of Medicine, National University Galway (NUIG), University Rd, Galway, Ireland

${ }^{\mathrm{f}}$ Fondazione Santa Lucia, Via Ardeatina, 306, 00142 Roma, Italy

${ }^{g}$ Niccolò Cusano University, Rome, via Don Carlo Gnocchi, 3, 00166 Roma, Italy

${ }^{\mathrm{h}}$ Maccabi Healthcare Services, Hamered Street 27, 68125 Tel-Aviv, Israel

\section{Corresponding Author:}

Carlos Pérez López

Centro de Estudios para la Dependencia y la vida Autónoma (CETpD)

Universitat Politècnica de Catalunya (UPC)

Rambla de l'Exposició 59

08800 Vilanova i la Geltrú,

Spain

Email: carlos.perez-lopez@upc.edu

Phone: +34938967274 


\section{Abstract}

Background: After several years of treatment, patients with Parkinson's disease (PD) tend to have, as a side effect of the medication, dyskinesias. Close monitoring may benefit patients by enabling doctors to tailor a personalised medication regimen. Moreover, dyskinesia monitoring can help neurologists make more informed decisions in patient's care.

Objective: To design and validate an algorithm able to be embedded into a system that PD patients could wear during their activities of daily living with the purpose of registering the occurrence of dyskinesia in real conditions.

Materials and methods: Data from an accelerometer positioned in the waist are collected at the patient's home and are annotated by experienced clinicians. Data collection is divided into two parts: a main database gathered from 92 patients used to partially train and to evaluate the algorithms based on a leave-one-out approach and, on the other hand, a second database from 10 patients which have been used to also train a part of the detection algorithm.

Results: Results show that, depending on the severity and location of dyskinesia, specificities and sensitivities higher than $90 \%$ are achieved using a leave-one-out methodology. Although mild dyskinesias presented on the limbs are detected with $95 \%$ specificity and 39\% sensitivity, the most important types of dyskinesia (any strong dyskinesia and trunk mild dyskinesia) are assessed with 95\% specificity and 93\% sensitivity.

Conclusion: The presented algorithmic method and wearable device have been successfully validated in monitoring the occurrence of strong dyskinesias and mild trunk dyskinesias during activities of daily living.

Keywords: Inertial sensors, Support Vector Machine, Parkinson's disease, Dyskinesia, Ambulatory monitoring. 


\section{Introduction}

Parkinson's disease (PD) is a chronic and second most common neurodegenerative disorder, behind Alzheimer's disease. The pathology of the disease is characterised by an insufficient activity in the neurons that produce dopamine, one of the main neurotransmitters involved in the control of movement [1]. First appreciable symptoms in PD are those related to an altered movement control, such as tremor, rigidity or bradykinesia (slowness of movement). These symptoms are caused by a decrease in dopamine levels. Presently, there is no known drug proven to have disease modifying effect or capable of stopping the progression of PD. Current main treatments are based on symptom reduction and centred in substances that increase the concentration of dopamine. More specifically, current treatments replace dopamine through the use of its precursor, Levodopa (L-Dopa), or substances that increase the neurotransmitter activity by stimulating dopamine receptors [2]. The main drug used in the early stages of the disease is L-Dopa taken as oral pills. During the first years of the disease, patients use to respond to oral treatment with L-Dopa in a stable way, without appreciable motor symptoms for most of the time. However, with the advance of the disease, the duration and effectiveness of the medication is reduced and patients tend to have, as a side effect of the medication, dyskinesias. These treatment-related motor complications appear in approximately $30 \%$ of patients after 2 years of L-Dopa exposure and, once established, they are very difficult to treat and significantly contribute to overall disability and disease burden $[3,4]$. Dyskinesia is a movement disorder that consists of involuntary movements that mainly occur in the limbs, hands, trunk and lingual-facial-buccal musculature $[5,6]$. At the time that these complications occur, the neurologist should consider a therapeutic change as these motor complications cannot be handled by oral medications [4].

In the latter years, some research has focused on helping neurologists to make care decisions in an objective way. For example, in the work presented in [7], an algorithm that selects one of the following three treatment options for patients with advanced PD is reported: subcutaneous apomorphine infusion, Deep Brain Stimulation and intestinal infusion of L-Dopa. This algorithm is mainly based on the age of the patient and the presence of dyskinesia. The idea behind the pump-based treatments (subcutaneous apomorphine infusion and L-Dopa intestinal infusion) relies on the 
growing evidence that improvement in duration and severity of dyskinesia can be achieved through continuous drug administration [4]. Thus, the change of treatment in PD is being defined, mainly, on the appearance of dyskinesias since they drastically affect the quality of life of patients and they can be improved with appropriate treatments.

Therefore, the gathered evidence has proven that the accurate knowledge of the appearance of dyskinesias during activities of daily living is indispensable for the neurologist in order to improve advanced PD treatment. This information can help the professionals to make informed decisions, on the one hand, to change the administration mode of the medication (oral / pump) or, on the other hand, to select another treatment to the patient (e.g. L-Dopa vs agonists). Furthermore, having this data available in realtime would open up the possibility to the on-line control of the dosage administrated by infusion pumps according to the patients' motor fluctuations. An example of this hypothesis was tested under previous work, in which a proof of concept was performed, by remotely adjusting the apomorphine dose (administered by a modified apomorphine pump) based on the motor phase of the patient [8].

The ultimate goal of the work presented in this paper is to design and validate an algorithm that can be embedded into a system that PD patients could wear during their activities of daily living with the purpose of registering the occurrence of dyskinesias in real conditions. Given that the system is designed to be employed in real conditions, its comfort and usability are crucial since patients should wear it for several hours. In this sense, one should not forget that the final system, in which these algorithms will be embedded, must be manipulated by PD patients who are generally aged over 65 and, additionally, the system must be continuously worn during waking hours (between 8 and 12 hours a day). These conditions, marked by patient's age, usage circumstances and PD symptoms, led us to make the system, and its method of placement in the body, to be very comfortable and very easy to use. To this end, as presented in the paper, the system is restricted to be a single device that unites sensor, processing and communication modules together.

The rest of the paper is organised as follows. The next section is devoted to describing the background and related work on dyskinesia detection. Third section 
presents the employed methods, including the data collection and the signal processing. Then, results are provided and finally, discussion and conclusions are presented.

\section{Related work}

Two types of dyskinesias are distinguished according to their clinical phenomenology: choreic and dystonic $[3,6]$. A choreic movement is an involuntary spasmodic twitching or jerking in muscles with no purpose. A dystonic movement involves contractions in muscle groups provoking abnormal postures. There are different classifications of dyskinesias but, when referring to PD, the most widespread classification is based on the timing of their appearance regarding the medication effect. According to this classification we can find, as Table 1 shows, three types: "off-phase dystonia", "diphasic dyskinesia" and "peak dose dyskinesia" [3]. The first type, off-phase dystonia, arises during off periods and is generally related to akinesia. Off periods are related to low dopamine levels in blood and, therefore, are often associated with periods in which the medication is not making the desired effect or when the plasma level of the active drug is low. Diphasic dyskinesias, instead, are associated to the onset and the end of dose, i.e. rise and fall of dopaminergic levels. Finally, peak dose dyskinesias are correlated with maximal plateau dopaminergic plasma levels. Each one of the three types of dyskinesia is associated with a specific set of involuntary movements, defining the way in which they are observed. First, peak-dose dyskinesias generally affect the trunk and consist of choreic form movements. Diphasic dyskinesias take the form of dystonic ballistic movements that are sometimes painful, although they commonly affect the lower limbs, in contrast to peak-dose dyskinesias that typically involve the upper limbs [4,9]. Peak-dose dyskinesias are the most common and frequent type of dopaminergic-induced dyskinesias [9]. Consequently, they are the most interesting type, from a clinical point of view, in order to assess the adverse effects of specific medications [10]. 


\begin{tabular}{|c|c|c|c|}
\hline $\begin{array}{l}\text { Classification } \\
\text { in PD }\end{array}$ & Type & Timing of the appearance & $\begin{array}{l}\text { Part of the } \\
\text { body } \\
\text { (commonly) }\end{array}$ \\
\hline $\begin{array}{l}\text { off-period } \\
\text { dyskinesia }\end{array}$ & Dystonic & $\begin{array}{c}\text { During off periods } \\
\text { (Low blood concentration of dopamine) }\end{array}$ & Distal extremities \\
\hline $\begin{array}{l}\text { Diphasic } \\
\text { dyskinesia }\end{array}$ & $\begin{array}{l}\text { Dystonic } \\
\text { and } \\
\text { Choreic }\end{array}$ & $\begin{array}{c}\text { End of dose } \\
\text { (Change in the blood concentration of } \\
\text { dopamine) }\end{array}$ & Lower limbs \\
\hline $\begin{array}{l}\text { Peak-dose } \\
\text { dyskinesia }\end{array}$ & Choreic & $\begin{array}{c}\text { During on periods } \\
\text { (High blood concentration of dopamine) }\end{array}$ & $\begin{array}{l}\text { Trunk and upper } \\
\text { limbs }\end{array}$ \\
\hline
\end{tabular}

Table 1: Dyskinesia classification based on appearance timing.

Research on automatic detection of choreic dopaminergic-induced dyskinesia relies on body-worn sensors based on Micro-Electro-Mechanical Systems (MEMS) technology, given that MEMS provide miniaturised wearable devices capable of being employed in unobtrusive human movement monitoring applications [11]. Main sensors that have been employed in choreic dyskinesia detection are accelerometers, gyroscopes, magnetometers and electrogoniometers [12-18]. Among them, accelerometers are the most widely used sensors to detect dyskinesia. They have been located in different parts of the body, such as trunk, limbs, or head [6]. Locations that have been mostly used are arms, legs and trunk [12-14] when more than one device is used in data acquisition. However, when just one single accelerometer is utilised the choice of localisation differs, i.e. shoulder [15] or waist [16].

Choreic dyskinesia assessment methods are commonly composed of the following steps. First, raw signals acquired from patients' movement are segmented into time windows and signals contained in each time window are characterised through some specific features. Then, these features are used as the input to a machine learning technique. It should be noted that some studies have only evaluated feature significance based on statistical tests [13,15,19-21], which does not facilitate an automatic assessment tool. The approaches found in the literature aiming to automatically assess dyskinesia are described in the rest of this section.

One of the most widely used ways to characterise MEMS sensor signals to detect dyskinesia is based on frequency analysis of the acquired data [12-17,21]. In these studies, signals were characterised by a Short Time Fourier Transform with the aim of representing signals in a frequency domain. Manson et al. proved that choreic dyskinesias increase the power spectrum density of accelerometer signals in the 
frequency band between 1-3 Hz [15]. Keijsers et al. [12] employed the frequency and amplitude of the signals to characterise them in the detection of dyskinesia. A similar approach was carried out in other studies $[16,17]$. Power spectrum analysis was also used in [13,21], however, this time on a larger bandwidth that reached $8 \mathrm{~Hz}$. In another recent paper [14], the signal energy within $2-5$ and $5-10 \mathrm{~Hz}$ frequency bands and entropy of the frequency spectrum were employed. Finally, high frequency energy content, frequency domain entropy and a five-bin histogram representation of the spectral contents over all three axes of the accelerometer have also been employed [17]. Besides the frequency based features, signal characterisation has also been done through minimum, maximum, mean and standard deviation values of the signals, RMS, entropies and correlations [14,17-20]. These features have been applied to other signals derived from the ones provided by the sensors, such as magnitude signal or velocity and displacement, obtained by single or double integration of the acceleration signals, respectively.

Recent studies on automatic dopaminergic-induced dyskinesias assessment have, however, two main limitations. First, the amount of signals obtained from patients is limited and most of them were collected from less than 20 PD patients [12,14-18]. Thus, as far as the authors know, there is no precedent work in which dopaminergicinduced dyskinesias were assessed in the daily life environment of a relevant sample of PD patients. In addition, it is unknown if these studies analysed any of the usability restrictions previously described in this article, since the great majority of them use some sensors distributed on the body [12-14,17,18,20,22]. As far as authors know, the only paper, apart from the previously submitted by authors [16], that uses a single sensor for dyskinesia assessment is operating from the shoulder [15], which is a position contrary to the restrictions that have been presented so far. In contrast to these previous approaches, this paper presents a new methodology to detect choreic dopaminergicinduced dyskinesias based on the signals from a single accelerometer positioned in the waist. 


\section{Methods}

This section describes the methods used to validate the device and algorithms for the assessment of choreic dyskinesia. The section is organised as follows. First, the authors describe the selection of the sensor position and the related implications. In the next section, the inclusion criteria and descriptive data of the patients who participated are presented. In the data collection part, the data acquisition methodology is explained. In the last sections, the signal processing and the evaluation techniques are detailed.

\subsection{Sensor position}

In the design and generation of the algorithms to monitor dyskinesia, it is essential to determine the context and conditions in which they will be used. In our case, the algorithm should be able to evaluate the choreic dyskinesia and be embedded in one or more devices that patients wear during their activities of daily living. According to this reasoning, two necessities lead and constrict the design and the specification of the device: the number and position of movement sensors.

In this work we face a compromise between the feasibility of capturing and recognizing a motor symptom and, on the other hand, the discomfort that can cause to the user wearing a sensor in certain positions or simply having to wear several sensors. Regarding the final wearable system, which is expected to embed the algorithm presented in this paper, it is essential not to be overly complex. Thus, in this paper we discard any system implementation that requires multiple sensors and we have focused the study in conducting an analysis with a single sensor.

In this sense, some studies like those of Yang et al. and Gjoreski et al. [23,24] argue that placing an accelerometer on the waist is interesting from a mechanical point of view, because of the closeness and solidarity with the centre of mass of the human body, providing good ergonomics for the patient. In addition, on the same line Mathie et al. [25] performed a survey where volunteers chose the side of the waist, above the anterior superior iliac spine, as the most suitable place to carry a small inertial sensor.

Given this reasoning and the restriction on the position and the number of sensors, in the work presented in this paper it has been considered that a single sensor must be used and, moreover, the side of the waist must be its position. All choreic dyskinesias are reflected, in greater or lesser extent, in the trunk (as most movements, 
even in the extremities, provoke some movement in the trunk) and, among the different trunk locations in which a sensor could be located, the waist is considered the optimal place. In this sense, it is considered that the waist is the most suitable place in the body that can be used to capture most of choreic dyskinesias using a single sensor. This consideration is a very hard restriction that appears from a usability point of view. The biggest problem is that, although this position enables the detection of dyskinesias appearing in almost any part of the body, some precision is lost in the degree of severity, depending on the area of occurrence; e.g. the sensed severity of an arm dyskinesia would be not comparable with the severity sensed from a similar dyskinesia in the trunk. This means that the detector will present greater sensitivity for trunk dyskinesia than for dyskinesias that occur in distal extremities.

In any case, sensor placement at the waist provides a good resolution for almost any choreic dyskinesia, those related to peak dose dyskinesias, which are the most interesting to detect from a clinical point of view. It is for this reason that, in the results section, the behaviour of the algorithm is presented in terms of the part of the body that the dyskinesia appears. However, the authors are aware of the trade-off and we prioritised usability and viability of the final system against a minimum reduction of the device resolution in certain types of dyskinesias.

\subsection{Participants}

An international multicentre study was planned to gather the inertial signals and validate the developed algorithms. The study took place from 2012 through 2014 and was coordinated by four different clinical settings: Centro Médico Teknon (Spain), National University of Ireland, Galway (Ireland), Fondazione Santa Lucia (Italy) and Maccabi Healthcare Services (Israel).

We included patients aged between 50 and 75 years, with idiopathic PD, according to the criteria of the UK PD Society Brain Bank [26]. All patients were in a moderate-severe phase (Hoehn and Yahr stage greater than or equal to 2.5 in their on state) and had motor fluctuations with bradykinesia, freezing of gait or dyskinesia. Table 2 presents the sociodemographic data and the distribution of the UPDRS scores, also the distribution of the patients' scores for dyskinesia are included. In Figure 1, a 
view of the distribution of patients according to the severity of dyskinesia, from the point of view of the sum of the scores of all items related to dyskinesia, is presented.

Patients with other health problems that hamper physical activity and patients with dementia (DSM-IV-TR criteria) or neuropsychiatric disorders were excluded. The study protocol was approved by the local Ethical Committee in each country. All participants provided informed consent prior to their inclusion in the study.

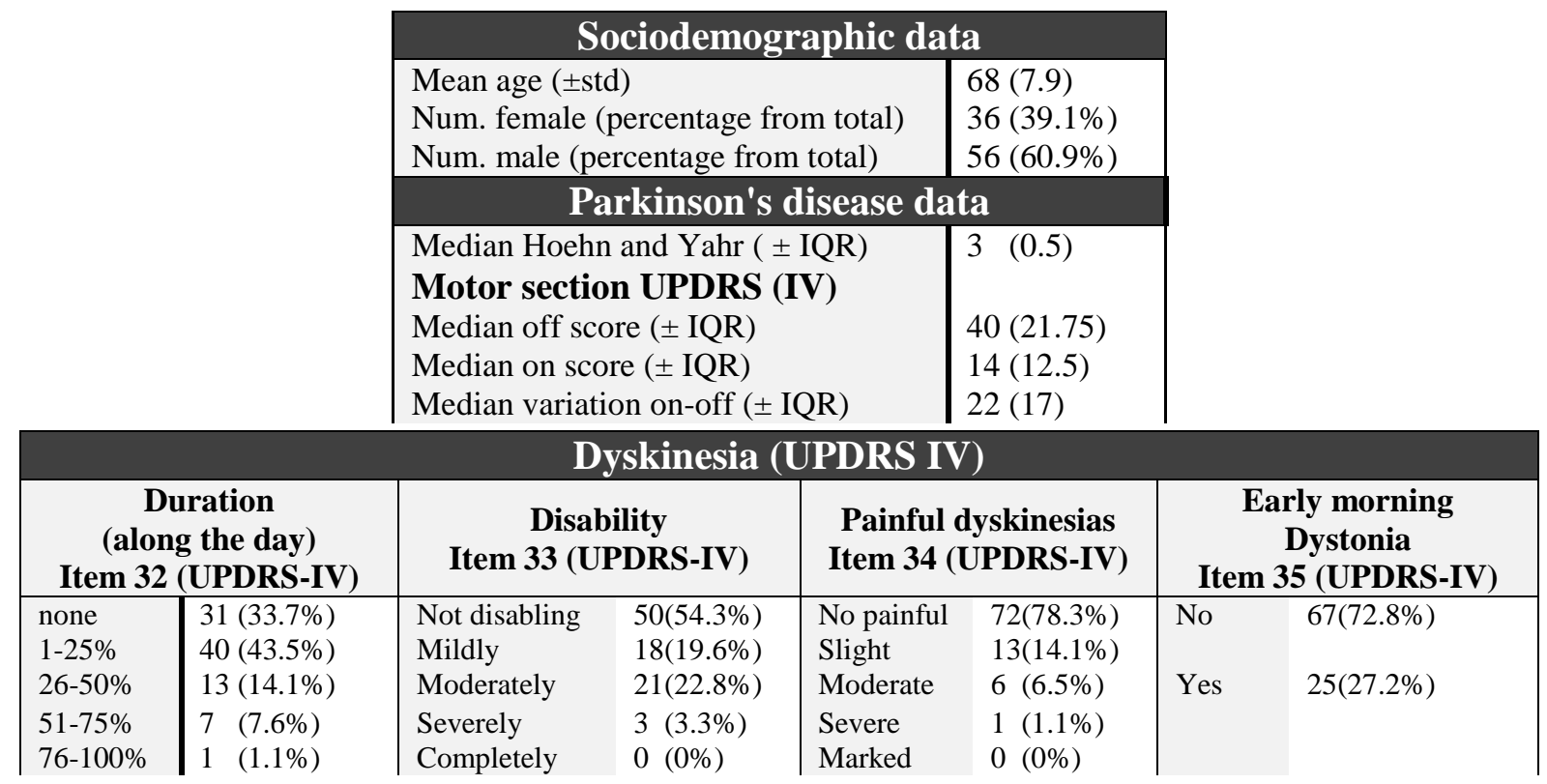

Table 2: Sociodemographic data, distribution of the UPDRS IV score, and the distribution of the scores for dyskinesia items of the patients included in the study, where std is the standard deviation and IQR is the interquartile range.

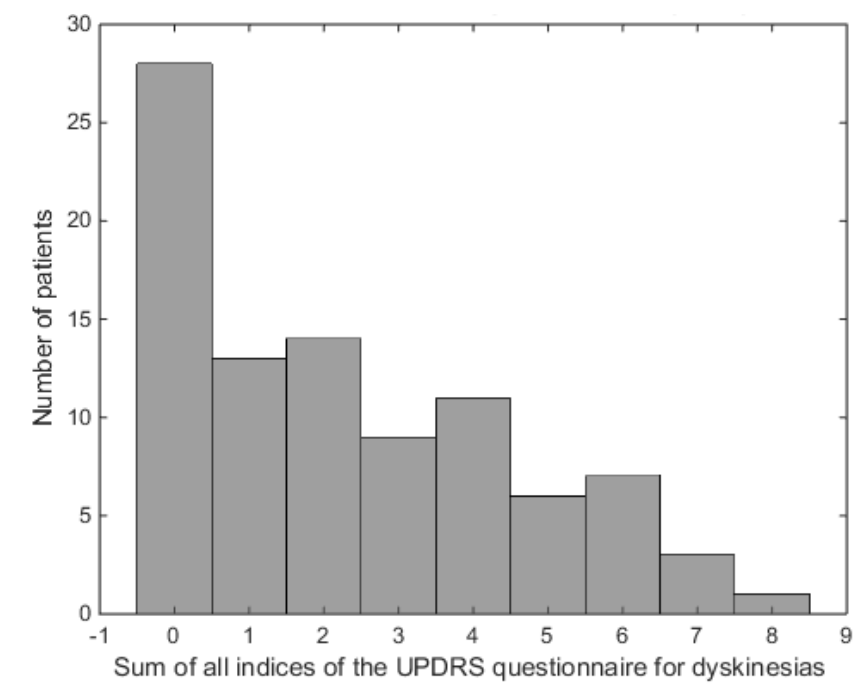

Figure 1: Distribution of patients according to the sum of UPDRS IV dyskinesia items $(32,33,34,35)$ 


\subsection{Data collection}

Data collection is divided into two parts: on the one hand, a main database which was built with 92 patients was used to partially train and to evaluate the algorithms and, on the other hand, a second database obtained from 10 patients [16]. The second database has been employed to train the window-based analysis of the algorithm, while the first one has been used to train the minute-level (meta-analysis) and validate the method based on a leave-one-out methodology. Both databases are presented in the following subsections.

\subsubsection{Main database of inertial signals}

Data collection was performed at patients' home in order to gather inertial signals similar to those that would be obtained in the daily life of PD patients. The experiment was also designed with the aim of ensuring that the different motors states of each patient were captured; also, an additional aim was to obtain representative data of patients' motor fluctuations. With the purpose of guaranteeing these points, it was used a widely extended method employed in clinical experiments in order to assess the worst symptoms of the disease by either removing or reducing the patients' antiparkinsonian medication, prior to the experiment, so as to force a low plasma level of dopamine. The study consisted on a series of tests in which the patient performed some activities that, although they were guided, their execution was free (e.g. patients were asked to show their home to the researchers), aiming to capture how motor symptoms (bradykinesia, dyskinesia, freezing of gait, etc.) appear in a real environment when the patients present different plasma dopamine levels. The environment has been shown to be very important in PD since, for instance, freezing of gait frequency is commonly reduced during laboratory evaluations [27]. In the data collection, the gold standard was generated from a video recording that was used for labelling the signal (off-line) by a trained expert, who took charge and carried out the experimentation, as it is explained below.

The data collection was performed twice, before and after the intake of the medication. Thus, in the morning, after removing the last antiparkinsonian drug intake of the previous day, specific tests for off state were performed, being its execution free. 
When the tests were finished the patient proceeded to take his/her regular antiparkinsonian medication and the researchers waited for the drug to take effect (for a minimum of one hour). Then, the researchers proceeded to perform the specific tests.

A list of the activities performed by each patient during each motor state is shown in Table 3 (more details can be found in [28]). All data were collected by research assistants who had been specifically trained in the study procedures and administration of the corresponding questionnaires, on the basis of common instructions and clinical guidelines. The same researchers who recorded the signals also labelled them according to the video and symptoms presented.

\begin{tabular}{|c|c|c|c|c|}
\hline \multicolumn{5}{|c|}{ Tests before the medication intake } \\
\hline UPDRS & $\begin{array}{c}\text { Indoors walking } \\
\text { test }\end{array}$ & $\begin{array}{c}\text { FoG provocation } \\
\text { test }\end{array}$ & $\begin{array}{c}\text { Outdoors } \\
\text { walking test }\end{array}$ & $\begin{array}{c}\text { Intake of } \\
\text { medication }\end{array}$ \\
\hline \multicolumn{5}{|c|}{ Tests after the medication intake } \\
\hline UPDRS & $\begin{array}{c}\text { Indoors walking } \\
\text { test }\end{array}$ & $\begin{array}{c}\text { Outdoors walking } \\
\text { test }\end{array}$ & Dyskinesia test & $\begin{array}{c}\text { False positive } \\
\text { test }\end{array}$ \\
\hline
\end{tabular}

Table 3: List of tests performed by patients in each motor state

A summary of the activities carried out during the data collection is presented below:

Indoors walking test: the patient started sitting on a chair in the living room. Then, he/she stood up and showed his/her house to the researchers, showing each room and explaining what the room is for, just like if the house was being sold.

Outdoors walking test: whenever it was possible, the patient went for a 10 to 15 minute walk around the neighbourhood.

FoG provocation test: the test started with the patient sitting on a chair, then he/she stood up and walked through a door or passed through a narrow place, afterwards the patient performed a turn of 180 degrees and turned back to the chair where he/she was suggested to sit. The test was performed 10 times.

Dyskinesia test: this test was performed only when the researcher detected that dyskinesia appeared and it was performed only once in the entire data capture. It started with the patient sitting on a chair, then he/she stood up, and stayed still for 1 minute, 
afterwards he/she sat down again and stayed at rest for another minute. This test allowed the researchers to capture the dyskinesia in controlled conditions, in contrast to the remaining activities, in which dyskinesia may be captured while performing other activities.

False Positive Test: In this test, the patient was asked to perform various activities of daily living that are likely to be confused with motor symptoms. The first test started with the patient sitting on a chair in the kitchen. The patient was invited to stand up, and to walk from the kitchen to the furthest room in the house, carrying a full glass of water. Then, the patient was invited to perform the following activities: to brush the teeth, to shake a deodorant bottle, to erase something with a rubber, to type on a computer, to wipe the glasses or the furniture and to wash a glass of water.

\begin{tabular}{lc|ccc} 
& $\begin{array}{c}\text { TOTAL } \\
\text { (minutes) }\end{array}$ & $\begin{array}{c}\text { After } \\
\text { medication } \\
\text { (minutes) }\end{array}$ & $\begin{array}{c}\text { Intermediate } \\
\text { (minutes) }\end{array}$ & $\begin{array}{c}\text { Before } \\
\text { medication } \\
\text { (minutes) }\end{array}$ \\
Dyskinesia & $\mathbf{4 0 1 . 2 3}$ & 355.48 & 17.57 & 28.18 \\
Bradykinesia & $\mathbf{8 6 5 . 5 8}$ & 50.14 & 25.37 & 790.07 \\
Tremor & $\mathbf{3 3 3 . 7 3}$ & 94.18 & 15.29 & 224.26 \\
FoG & $\mathbf{1 4 0 . 5 2}$ & 20.55 & 7.18 & 112.79 \\
\hline Symptom-free & $\mathbf{1 7 6 1 . 4 2}$ & 1377.42 & 63.2 & 320.8 \\
\hline
\end{tabular}

Table 4: Minutes captured and labelled divided by state and symptoms

Signals employed in this work were gathered by means of the 9x2 inertial measurement unit [29] located at the waist near the iliac crest. This waist device is composed of an accelerometer, a gyroscope, a magnetometer, a microcontroller, a Bluetooth communication module and a memory storage unit. The 9x2 maximum sample data rate is $200 \mathrm{~Hz}$ and data is saved in a SD memory card. The approach presented in this paper only employs the accelerometer signals, discarding measurements from gyroscopes and magnetometers. In the case of gyroscopes, given the frequency nature of the proposed method these sensors do not provide relevant information and, in addition, their power consumption is much higher and they commonly present large drifts due to the manufacturing process and ambient temperature. On the other hand, magnetometers present a high sensitivity to magnetic disturbances. It is for these reasons that the method presented in the next sections is based only on accelerometer measurements. 
Finally, through the video recordings, the same clinicians who recorded the signals also labelled them with the symptoms of the patient. Regarding choreic dyskinesias, the signals are labelled depending on the area of occurrence (head, hand / arm, foot / leg, trunk) and are also classified into two groups of severity (weak or strong) [28]. ¡Error! No se encuentra el origen de la referencia. presents a summary of the overall minutes of captured signals and their labelling.

\subsubsection{Secondary database of inertial signals}

A second dataset, that has been employed to partially train the algorithms, included a total of 10 PD patients. This study was carried out during 2008 and 2009, with patients between 49 and 82 years of age, living in the Barcelona area, who had been diagnosed to have idiopathic PD according to the criteria of the Brain Bank, London [26]. Only patients with mild or moderate stage of the disease and motor fluctuations were included in the study. For security reasons, patients with implanted electronic devices were excluded.

Patients performed various activities while an inertial sensor located at their waist registered acceleration. Signals employed in this work were gathered by means of the same 9x2 system presented previously [29]. The protocol included activities in the laboratory and outdoors. Laboratory activities comprised walking in a straight line, walking over an inclined plane, carrying a heavy object, setting a table and going upstairs and downstairs. The outside protocol consisted of walking for, at least, 15 minutes. Patients that had motor fluctuations repeated the experiment, excluding the outdoors protocol, in off state, which was induced by avoiding the first morning intake of medication. The experimental protocol was approved by the local Ethics Review Committee.

\subsection{Signal processing}

As described in the previous sections, the sensor position is a fundamental choice in the design of the algorithms that perform the signal processing associated with the detection. This section continues with the premise of using a single sensor positioned at the waist, as justified in the previous sections. Figure 2 shows the accelerometer signals 
captured from the previously presented waist device worn by a patient with and without dyskinesia while sitting, standing and walking. It is observed that when the patient does not present dyskinesia and is sitting, there are not relevant harmonics in the signal while, when the patient has dyskinesia, harmonics in the lower frequencies appear. However, it is observed that power spectrum of the lower frequencies is not only increased during dyskinesia but also during other activities, such as walking or climbing stairs, as described in [15].

Regarding these observations, the authors developed a signal processing method previously presented in [16]. This preceding method considers the power spectrum of the frequency band composed of 1 to $4 \mathrm{~Hz}$ to detect dyskinesia, as long as higher frequencies ( 8 to $20 \mathrm{~Hz}$ ) do not have a high power spectrum, which correspond to false positives like walking or climbing stairs. The previously presented method, however, does not consider posture transitions (PT), which, as Figure 2 shows, also increase the power spectrum of the lower frequencies. In the current paper, the signal processing employed is an improvement of the one presented in [16], with the aim of, mainly, deal with PT, although other minor enhancements are also performed.
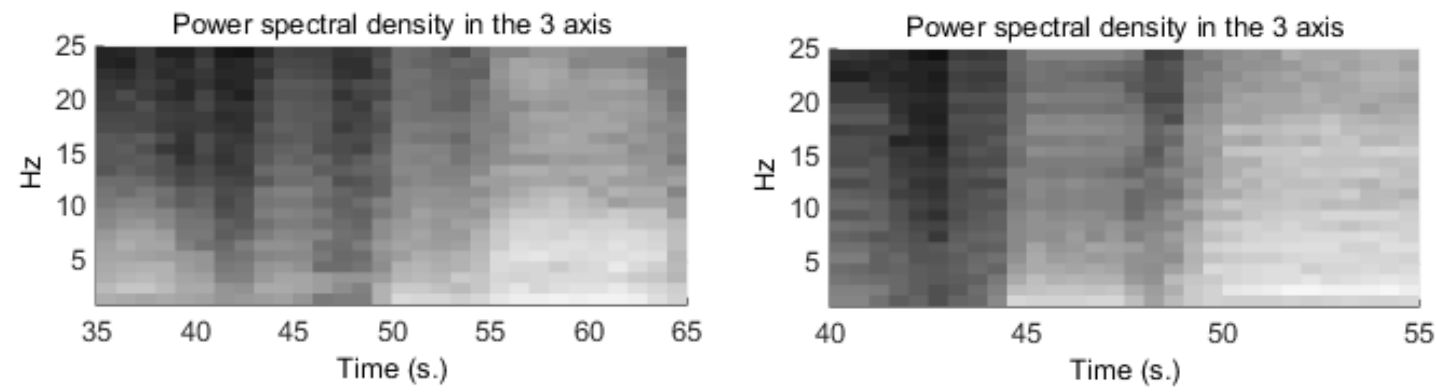

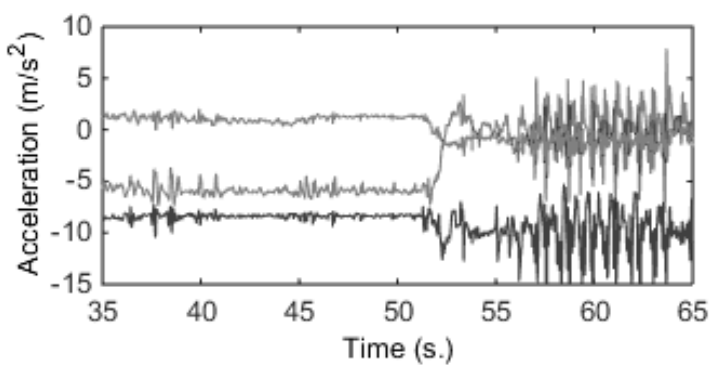

(a)

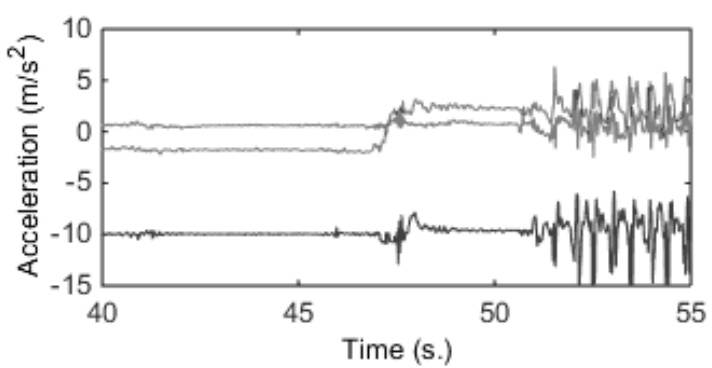

(b)

Figure 2: On the right (b), signal from a patient without dyskinesia. Patient sat during the time period 40-47 s., stood (47-52) and walked (52-55). On the left (a), signal from another patient with dyskinesia. Low frequency and amplitude harmonics are observed for dyskinetic patient. Patient remained sat (until 51), stood (51-56) and then walked. Higher power density content is shown with white colour and lower power density content with black colour. 


\section{Step 1: Window-based analysis}

The signal processing employed to assess the presence of dyskinesia is based on splitting the spectra into three different bands:

- Dyskinetic band. A high power spectral density in this band is an indicator that either the patient has dyskinesia or the patient is walking or going up/down stairs. The dyskinesia band is considered to cover the $(0.68,4] \mathrm{Hz}$ range, where $0.68 \mathrm{~Hz}$ is considered to be the upper limit of PT band [30]. Dyskinesia band covers until $4 \mathrm{~Hz}$ since, although other studies consider $8 \mathrm{~Hz}$ to be the limit, frequencies higher than $4 \mathrm{~Hz}$ correspond to tremor according to a consensus of the Movement Disorders Society [31].

- Non-dyskinetic band is considered to be $[8,20] \mathrm{Hz}$. This band will enable us to know whether an increase in the dyskinetic band is due to dyskinesia appearance or due to walking (or similar activities). Given that walking has the $99 \%$ of its power spectral density below $20 \mathrm{~Hz}$ [32], $20 \mathrm{~Hz}$ is considered as the upper limit of the non-dyskinetic band. The lower limit of the band is provided by the maximum frequencies obtained in some dyskinesia research works [13]. In this way, harmonics in this non-dyskinetic band are used to determine the presence of gait or similar activities as the input of a machine learning classifier, as detailed below. A Support Vector Machine (SVM) is employed because of the complexity of detecting gait in PD patients through a waist sensor, especially when patients walk slowly. Moreover, the use of SVMs is adopted since gait detection is a bi-classification problem and SVMs have provided excellent results in similar tasks and because they commonly present good generalisation performance [33]. Furthermore, SVMs have been used in investigations with very similar characteristics and very good results, for example the work of Shyamal et al. [34].

- PT band is considered to be $(0,0.68] \mathrm{Hz}$ where $0.68 \mathrm{~Hz}$ is the upper limit of PT [30]. This band will enable us to know whether a PT has occurred.

Power spectrum in dyskinesia band is denoted as $P_{d}$ and spectral power in the PT band as $P_{P T}$. The amplitude of the harmonics of the non-dyskinetic band are represented by $h_{1}, \ldots, h_{k}$.

The minimum usable sample frequency $\left(F_{s}\right)$ has been determined by the maximum frequency of interest, which in this case is $20 \mathrm{~Hz}$ from the walking band. In 
consequence, $F_{s}$ has been set to $40 \mathrm{~Hz}$, implying that signals collected were re-sampled from $200 \mathrm{~Hz}$ to $40 \mathrm{~Hz}$ before applying the described algorithm. On the other hand, FFT, which has been employed to obtain the power spectra, requires a window whose length is a power of 2 in order to be executed in real-time with minimum resources. Possibilities taken into account are 32 (0.8 s.), 64 (1.6 s.), 128 (3.2 s.) and 256 (6.4 s.) samples. Given that $0.68 \mathrm{~Hz}$ is the minimum frequency for the dyskinetic band, the temporary window should be above $1.5 \mathrm{~s}$. in order to capture the "slowest" dyskinetic cycles. However, $1.6 \mathrm{~s}$ are not enough to capture the PT band so, consequently, FFT window length is set to 128 samples and $w$ value is set to 3.2 seconds.

Finally, the dyskinesia algorithm's output in a window $i$ is defined on these spectral power values according to:

$$
S_{i}= \begin{cases}1 \text { (Dyskinesia) } & P_{d}>d \wedge P_{P T}<d_{P T} \wedge f\left(h_{1}, \ldots, h_{k}\right)=1 \\ 0 \text { (No dyskinesia) }) & P_{d} \leq d \wedge P_{P T}<d_{P T} \wedge f\left(h_{1}, \ldots, h_{k}\right)=1 \\ U \text { (Unknown) } & P_{P T} \geq d_{P T} \vee f\left(h_{1}, \ldots, h_{k}\right)=-1\end{cases}
$$

where $d$ and $d_{P T}$ are the thresholds for dyskinetic and PT bands, respectively, $f\left(h_{1}, \ldots, h_{k}\right) \rightarrow\{1,-1\}$ is the decision function of the trained SVM and, additionally, provides value -1 when patient has walked, used stairs or performed a similar activity while, otherwise, provides value 1.

Parameters $d, d_{P T}$ and decision function $f$ have been set by means of specific procedures applied to the set of labelled signals collected from 10 patients, described in Section 3.3.2, and that correspond to a previous study [16]. The specific procedures employed to set their values are described below:

- First, function $f$ is set based on a set of vectors $\left\{\mathbf{p}_{1}, \ldots, \mathbf{p}_{n}\right\}, \mathbf{p}_{i}=\left[h_{1}, \ldots, h_{k}\right]$ obtained from several windows of the previously collected signals [16]. Each one of these vectors $\mathbf{p}_{i}$ is associated with a label $y_{i}=\{1,-1\}$ according to the labels given by clinicians to that window: $y_{i}=1$ corresponds to those windows labelled with walking or going up/down stairs and $y_{\mathrm{i}}=-1$ to the remaining windows. Dataset elements are denoted as $\left\{\left(\mathbf{p}_{1}, y_{1}\right), \ldots,\left(\mathbf{p}_{n}, y_{n}\right)\right\}$ and were employed to train a SVM with a radial basis function kernel: 


$$
\begin{aligned}
& \min _{\mathbf{w}, b, \xi} \frac{1}{2}\|\mathbf{w}\|_{2}^{2}+C \sum_{i=1}^{N} \xi_{i} \\
& \text { s.t. } \quad \xi_{i} \geq 0 \\
& \qquad y_{i}\left[K\left(\mathbf{w}, \mathbf{p}_{i}\right)+b\right] \geq 1-\xi_{i}
\end{aligned}
$$

where $K\left(\mathbf{w}, \mathbf{p}_{i}\right)=e^{\gamma \mid \mathbf{w}-\mathbf{p}_{i} \|_{2}^{2}}, b$ is the hyperplane bias, $\mathbf{w}$ is the hyperplane that separates both classes and $\xi_{i}$ are the slack variables. Parameters $C$ and $\gamma$ are determined as the values that maximise the accuracy among the values $10^{-2}, \ldots, 10^{2}$ in a 10 -fold CrossValidation [35]. The final form that $f$ gets in order to determine the output value for a new window represented by $\mathbf{p}$ is:

$$
f=\operatorname{sgn}\left(\sum_{i=1}^{l} y_{i} \alpha_{i} K\left(\mathbf{p}_{i}, \mathbf{p}\right)+b\right)
$$

where the set of alphas are the Lagrangian multipliers of the dual problem formulated by the SVM.

- Second, $d_{P T}$ was set also through a similar optimisation method applied to specific values obtained from the previous study [16]. In this case, the dataset was composed of $P_{P T}$ values computed from window signals that satisfied $f\left(h_{1}, \ldots, h_{k}\right)=-1$, that is, the patient neither walked nor went up/down stairs. A label was obtained for each $P_{P T}$ and consisted in whether the patient performed a PT or not in the corresponding window. Given the set of $P_{P T}$ values and their associated labels, a linear kernel $K\left(\mathbf{w}, \mathbf{p}_{i}\right)=\mathbf{w} \cdot \mathbf{p}_{i}$ was used to find the hyperplane that separates both classes based on Equation (4). In this case, given that $\mathbf{p}_{i}$ is actually a scalar, w value obtained from Equation (4) is also scalar. Thus, the value found through the optimisation process is used as $d_{P T}$. The obtained value is $d_{P T}=0.95$.

- Third, $d$ was set through the same method than $d_{P T}$ although, in this case, the dataset was composed of $P_{d}$ values computed from those windows that satisfied $P_{P T}<d_{P T}$ and $f\left(h_{1}, \ldots, h_{k}\right)=-1$ (windows in which the patient neither walked, went up/down stairs and did not performed a PT). The label consisted in the presence or absence of dyskinesia. The value obtained is $d=1.75$. 
This way, for activities such as walking, using stairs or PT, in which $f\left(h_{1}, \ldots, h_{k}\right)=-1$ or $P_{P T} \geq d_{P T}$ are satisfied, respectively, the algorithm's output will be unknown despite the patient has dyskinesia or not. This behaviour is actually associated with visual appreciation: when a patient has dyskinesia and walks, commonly it cannot be distinguished in the waist since walking hides the involuntary movement.

\section{Step 2: Meta-analysis}

Dyskinesia detection described by Equations (1) (2) and (3) enables us to know whether, in a given time window, a patient has dyskinesia. However, dyskinesia is a symptom which may last minutes. The most suitable window to examine the appearance of dyskinesia is, then, of several windows length. Thus, it is proposed to aggregate the output obtained in windows of $w$-seconds length overlapped at 50\%, similarly to [36], along a longer period of $T=60 \mathrm{~s}$. seconds. The algorithm provides, then, a single output obtained from the $2 T / w$ windows included in the 1-minute period according to:

- Dyskinesia if:

$$
\sum_{i=1}^{2 T / w} \frac{s_{i}}{n_{d}}>t_{p} \wedge \frac{w \cdot n_{d}}{2 T}>t_{c}
$$

- No dyskinesia if:

$$
\sum_{i=1}^{2 T / w} \frac{s_{i}}{n_{d}} \leq t_{p} \wedge \frac{w \cdot n_{d}}{2 T}>t_{c}
$$

- Unknown, otherwise, i.e.:

$$
\frac{w \cdot n_{d}}{2 T}<=t_{c}
$$

where $s_{1}, \ldots, s_{2 T / w}$ are the algorithm outputs represented in Equations (1) (2) and (3), being $s_{i}=1$ in case of dyskinesia detection and $s_{i}=0$ otherwise; $n_{d}$ is the number of time windows in which the condition $P_{P T} \geq d_{P T} \vee f\left(h_{1}, \ldots, h_{k}\right)=-1$ were not held (i.e. number of windows analysed), $t_{p}$ is the threshold which sets the dyskinesia detection rate needed to consider the $T$-seconds period as dyskinetic and $t_{c}$ sets the minimum amount of analysed windows in the period of $T$ seconds. 
The logic behind the algorithm consists in considering a $T$-seconds period to be dyskinetic if the summation in Equation (6) is greater than a certain threshold $t_{p}$. However, this value might not be enough by itself since it could not be reliable if the patient walked during most of the analysed period. Then, a confidence index is defined as $w \cdot n_{d} / 2 T$, which represents, from the total of $2 T / w$ analysed windows, the number of windows that were not rejected because of the condition $P_{P T} \geq d_{P T} \vee f\left(h_{1}, \ldots, h_{k}\right)=-1$. In this manner, a low confidence index is considered to provide an unreliable dyskinesia assessment and, then, an undetermined evaluation, since few windows could be analysed. Consequently, the confidence index is required to be greater than a certain threshold $t_{c}$.

Values for $t_{p}$ and $t_{c}$ were found based on an 'leave one-patient-out' approach on the signals collected from 92 patients. Accordingly, these two thresholds are set as those values that maximize $\sqrt{\text { Sensitivity.Specifity }}$ and that use at least half of the samples analysable based on the data from 91 patients and, then, the resulting values are evaluated with the data from the remaining patient. This process is repeated 92 times, one for each patient. More specifically, tested values for $t_{c}$ have been $(0,0.1,0.2, \ldots, 1)$ and for $t_{p}$ have been $(0.01,0.02, \ldots, 1)$. The evaluation of the obtained threshold values has been carried out by considering the same weight for each minute analysed.

\subsection{Evaluation}

Signals gathered are employed to evaluate the method presented above in nonoverlapped periods of $T=60$ seconds, resulting in sensitivity and specificity values. Each evaluated interval of $T=60$ seconds is required not to contain any sample labelled with any type of dyskinesia or to contain at least $N=F_{s} \cdot T / 2$ samples (i.e. $30 \mathrm{sec}$.) of the dyskinesia type under analysis. Depending on the output of the algorithm and the labels of the samples, each interval is considered a true/false positive/negative case. This way, signals labelled with at least $N$ samples of dyskinesia are then either false negatives (FN) or true positives (TP). On the other hand, those signals without any labelled dyskinesia are evaluated as true negatives $(\mathrm{TN})$ or false positives (FP)

The value used for $N$ is half the period of analysis, i.e. $F_{s} \cdot T / 2$, requiring at least 30 s. to be labelled as dyskinetic. Those periods in which the number of samples 
labelled with dyskinesia is not zero and lower than $30 \mathrm{~s}$. are considered to be indeterminate and not evaluated. Finally, it should be noted that minutes in which $\frac{w \cdot n_{d}}{2 T}<=t_{c}$ are considered like undetermined since patients mostly walked during them, as stated in previous sections. These restrictions reduce significantly the number of minutes used in the analysis but the veracity of the signals used for evaluation algorithm is ensured.

\section{Results and discussion}

Among the 92 patients included in the database, 35 presented some type of choreic dyskinesia. A total of 3228 minutes of labelled signals were collected, from which 401 minutes $(12 \%)$ were labelled with choreic dyskinesia.

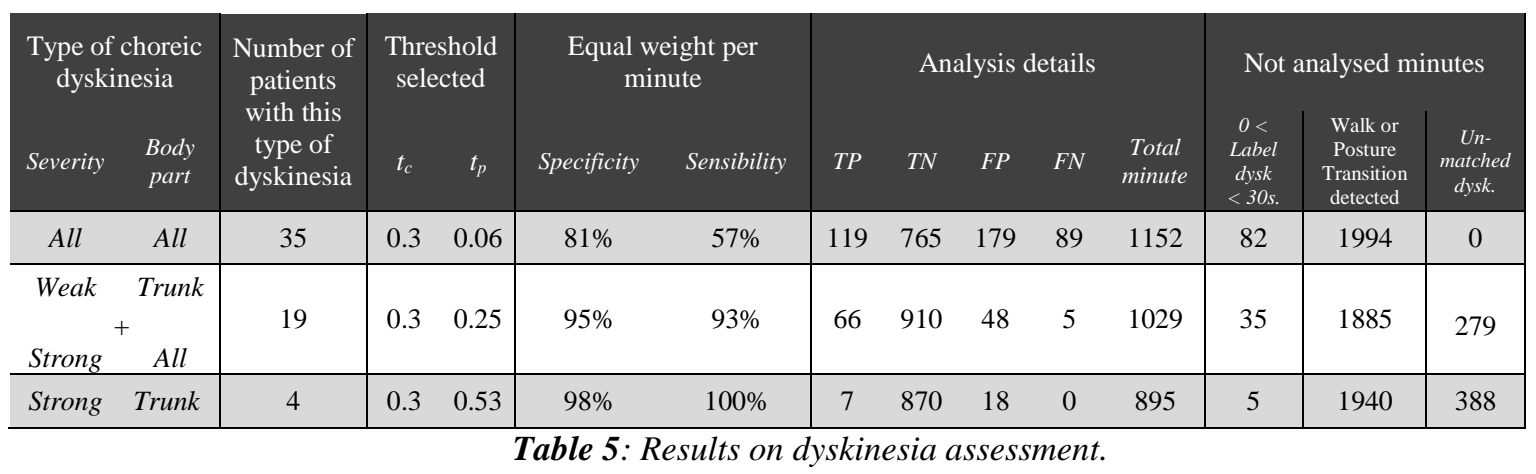

An overview of the results in choreic dyskinesia assessment based on a leaveone-out approach on the data collected from 92 patients is provided in Table 5. The table also presents, for each dyskinesia type analysed, the best values of $t_{p}$ and $t_{c}$ found in the leave-one-out procedure. Assessment results are presented according to the severity and the body part where the dyskinesia was presented, thus, the analysis is split into three sub-analysis. In the first sub-analysis (first row), results on detecting any choreic dyskinesia in any part of the body are presented. The second sub-analysis requires the algorithm to detect any strong dyskinesia from any part of the body adding weak dyskinesias in the trunk. Finally, in the third sub-analysis, the results correspond to evaluate the algorithm in detecting only strong trunk dyskinesias. In the third column of Table 5, the average sensitivity and specificity among all minutes is presented regarding the detection of each kind of dyskinesia. In the columns under "Analysis details”, TP, TN, FP and FN values are shown, as defined in previous Section 3.5. Also, 
the amount of minutes used in the analysis (summation of TP, TN, FP and FN) and the number of not analysed minutes are presented. It should be noted that, in the calculations of each row, those minutes labelled as dyskinesias, but that were not labelled with $30 \mathrm{~s}$. of the type of dyskinesia under analysis, were withdrawn from the analysis. The number of these minutes is presented in the last column as 'Unmatched dyskinesia'.

\begin{tabular}{|cc|c|}
\multicolumn{2}{|c|}{$\begin{array}{c}\text { Type of choreic } \\
\text { dyskinesia }\end{array}$} & $\begin{array}{c}\text { Minutes } \\
\text { labelled }\end{array}$ \\
\cline { 1 - 2 } Severity & $\begin{array}{c}\text { Body } \\
\text { part }\end{array}$ & \\
\cline { 1 - 2 } Weak & Trunk & 95 \\
\hline Strong & Trunk & 24 \\
\hline Weak & No-trunk & 306 \\
\hline Strong & No-trunk & 48 \\
\hline
\end{tabular}

Table 6: Total of minutes labelled on the database.

Results presented in Table 5 show that, depending on the severity and location of dyskinesia, specificities and sensitivities higher than $90 \%$ can be achieved using a leave-one-out methodology. Although mild dyskinesias presented on the limbs are detected with $95 \%$ specificity and 39\% sensitivity, the most important types of dyskinesia (any strong dyskinesia and trunk mild dyskinesia) are assessed with 95\% specificity and $93 \%$ sensitivity. If strong trunk dyskinesia is considered in an isolated way, they are detected with $98 \%$ and $100 \%$ of specificity and sensitivity, respectively. These results confirm that the algorithm is very sensitive to trunk dyskinesia, this fact is confirmed by the behaviour of the method which is very good for all strong and weak trunk dyskinesias; however, it presents a worse response when weak dyskinesias in the extremities are included in the analysis.

It should be noted that the different types of dyskinesia analysed and presented in Table 5 are presented in an aggregated order: the first one of the three types include the other two types (e.g., all dyskinesias with any severity includes the weak trunk dyskinesia and all the strong ones), in the same way than the second kind of dyskinesia analysed comprises the third one. Table 5, on the other hand, presents a decrease in the time analysed along with the more specific dyskinesia analysed. This occurs because the evaluation of more specific types of dyskinesia permits to evaluate fewer labelled 
episodes since those minutes which are labelled without the specific type of dyskinesia are not analysed. In Table 5, the number of unanalysed minutes is also relevant since patients were asked to walk in most activities of the protocol; however, in real life monitoring these walking minutes would be drastically reduced, increasing the analysis time.

Table 6 shows the number of labelled minutes of each type of dyskinesia divided into weak and strong, and trunk and no-trunk (distal). Note that this type of dyskinesias are not exclusive, for example, a minute of signal presenting weak trunk dyskinesia and, at the same time, weak dyskinesia in a hand can be found. On the other hand, it should be mentioned that, in this sum, dyskinesia labels are globally considered, including labelled dyskinesias while patients are walking, which are extracted from the analysis based on Equation (3).

\begin{tabular}{|c|c|c|c|c|c|}
\hline \multicolumn{2}{|c|}{$\begin{array}{l}\text { Type of choreic } \\
\text { dyskinesia }\end{array}$} & \multirow{2}{*}{$\begin{array}{l}\text { Num. of } \\
\text { patients with } \\
\text { this type of } \\
\text { choreic } \\
\text { dyskinesia }\end{array}$} & \multicolumn{2}{|c|}{$\begin{array}{l}\text { Equal weight per } \\
\text { minute }\end{array}$} & \multirow[b]{2}{*}{$\begin{array}{c}\text { Total } \\
\text { minutes }\end{array}$} \\
\hline Severity & $\begin{array}{l}\text { Body } \\
\text { part }\end{array}$ & & Specificity & Sensibility & \\
\hline Weak & Trunk & 16 & $95 \%$ & $78 \%$ & 953 \\
\hline Strong & Trunk & 4 & $95 \%$ & $100 \%$ & 895 \\
\hline Weak & No-trunk & 32 & $95 \%$ & $39 \%$ & 1110 \\
\hline Strong & No -trunk & 7 & $95 \%$ & $90 \%$ & 917 \\
\hline
\end{tabular}

Table 7: Results on dyskinesia assessment for the thresholds $t_{p}=0.25$ and $t_{c}=0.3$.

In addition, in order to evaluate the performance of the algorithm against various types and severities of dyskinesias, a further analysis has been done. Due to the particularity of the measurement position, a low sensitivity for weak dyskinesias of the distal extremities is expected. For this reason, the most interesting case presented in Table 5 is the row that jointly evaluates weak trunk with strong all dyskinesias (middle row). According to this premise, Table 7 shows the results of the algorithm on each type of dyskinesia divided into weak/strong and trunk/no-trunk (distal) for the optimal thresholds 0.25 and 0.3 for $t_{p}$ and $t_{c}$, respectively, found for the mentioned case (middle row of Table 5). Note that this type of dyskinesias are not exclusive so, for instance, a patient with weak trunk dyskinesia could be found at the same time with weak dyskinesia in a hand. Results show how the algorithm presented is highly sensitive to dyskinesias labelled as strong. We also find that a high sensitivity is 
maintained for mild trunk dyskinesia, whereas the sensitivity drops in assessing weak dyskinesias in distal extremities. Notably, in both Table 5 and Table 7, the method presents a high specificity, meaning that confidence when the algorithm output diagnoses dyskinesia is very high.

Comparing the results of the presented algorithm to those obtained in previous works, it is observed that, first, the performance obtained on detecting the presence or absence of dopaminergic-induced dyskinesia in 1-minute periods by Keijsers et al. through a single sensor located on the trunk provided $82 \%$ of accuracy [12]. In the approach presented in this paper, a $95 \%$ of accuracy on trunk dyskinesia and any severe dyskinesia from any part of the body is obtained, surpassing by more than $10 \%$ the previous results. Results from Keijsers et al. were obtained following a 'leave onepatient-out' approach with 12 patients. In contrast, the method proposed in this paper has been tested on data from 92 patients following the same 'leave one-patient-out' approach. Results from Tsipouras et al. [14] achieved a 93\% of accuracy through the same leave-one-patient out approach. However, these results were obtained based on 6 sensors and with signals from 16 volunteers. Finally, the study from Chelaru et al. [18] provided an accuracy of $100 \%$ on dopaminergic-induced dyskinesia detection. Nonetheless, signals were collected with patients standing and refraining from making voluntary movements.

In summary, results obtained by the presented approach either outperform previous studies or are similar. However, it should be noted that signals used in this study to evaluate the proposed method posed a higher challenge given that they come from a single sensor and they belong to a population of 92 PD patients. Thus, the device and algorithm presented here, as far as authors are aware, and compared to the previously presented works, is the only set-up that meets the specifications raised: an algorithm to evaluate dyskinesias that can be embedded in a single device worn in the waist in the daily life environment of patients.

\section{Conclusion}

The ultimate goal of this work is to develop the necessary algorithms to evaluate the occurrence of dyskinesias in a patient while performing the activities of daily life. Moreover, these algorithms must be embedded in hardware with some physical and computational restrictions. These restrictions are not only related with the memory and 
computing resources, but also with the position and location of the sensor. These restrictions, as shown in this paper, affect the process of development, generation and accuracy of the algorithm. This type of device would provide relevant information to the clinicians in tailoring treatment and, furthermore, it is now available.

The algorithm presented in this paper has been evaluated on a database of signals gathered in the daily life environment of 92 PD patients, under a multicenter study that took place in 4 different countries. The approach provides specificities and sensitivities above 90\%, showing that choreic dyskinesia is able to be monitored through a single sensor during daily life of patients. However, the results also show that the restrictions imposed on the final system heavily penalise its accuracy for some types of dyskinesias, since weak dyskinesias in the distal extremities are not correctly recognised. The consequence of these constraints, based on the physical limitations of the users and the usability characteristics of the system, result in a trade-off between sensitivity in detecting weak dyskinesias on the distal limbs and the specificity of the method. From the beginning of this work, it has been taken into account that the ultimate objective of the system is to provide information on the dyskinetic states, which should be considered when the neurologists are making a decision in the treatment for patients with advanced PD. Thus, the most important state to be detected is severe choreic dyskinesia, which is detected with excellent sensitivity and specificity with the current system.

From a clinical point of view, the correct identification of dyskinesia is a very significant issue for patients with PD since it significantly affects patient's functional autonomy and is associated to a greater risk of falls. Moreover, dyskinesia detection, in addition to other motor symptoms detection, relies on objective tools, such as the proposed in this paper, that enable treatment adjustment of PD patients. The information that these systems are capable of providing to neurologists will contribute to a better understanding of PD and, combined with other tools such as behavioural patterns, will contribute to timely intervention and will prevent patient deterioration based on fine adjustments of PD treatment.

\section{Acknowledgements}


This work has been performed in the framework of the FP7 project REMPARK ICT287677, which is funded by the European Community. The author(s) would like to acknowledge the contributions of their colleagues from REMPARK Consortium (http://www.rempark.eu). The authors would like to thank all patients who participated in the study.

\section{Bibliography}

[1] J. Jankovic, Parkinson's disease: clinical features and diagnosis, Journal of Neurolology, Neurosurgery and Psychiatry. 79 (2008) 368-376. doi:10.1136/jnnp.2007.131045.

[2] E. Chávez-León, M.P. Ontiveros-Uribe, J.D. Carrillo-Ruiz, La enfermedad de Parkinson: neurología para psiquiatras, Salud Mental. 36 (2013) 315-324.

[3] G. Fabbrini, J.M. Brotchie, F. Grandas, M. Nomoto, C.G. Goetz, Levodopa-induced dyskinesias, Movement Disorders. 22 (2007) 1379-1389.

[4] G. Abbruzzese, P. Barone, U. Bonuccelli, L. Lopiano, A. Antonini, Continuous intestinal infusion of levodopa/carbidopa in advanced Parkinson's disease: efficacy, safety and patient selection, Functional Neurology. 27 (2012) 147.

[5] E. Santini, E. Valjent, G. Fisone, Parkinson's disease: Levodopa-induced dyskinesia and signal transduction, Federation of European Biochemical Societies Journal. 275 (2008) 1392-1399.

[6] M.R. Luquin, O. Scipioni, J. Vaamonde, O. Gershanik, J.A. Obeso, Levodopa-induced dyskinesias in Parkinson's disease: Clinical and pharmacological classification, Movement Disorders. 7 (1992) 117-124.

[7] A. Antonini, E. Tolosa, Apomorphine and levodopa infusion therapies for advanced Parkinson's disease: selection criteria and patient management, Expert Review of Neurotherapeutics. 9 (2009) 859-867. doi:10.1586/ern.09.48.

[8] A. Rodríguez-Molinero, D.A. Pérez-Martínez, C. Gálvez-Barrón, J. Hernández-Vara, J.C. Martínez-Castrillo, R. Álvarez, et al., Remote control of apomorphine infusion rate in Parkinson's disease: Real-time dose variations according to the patients' motor state. A proof of concept, Parkinsonism \& Related Disorders (2015). doi:10.1016/j.parkreldis.2015.04.030.

[9] R. Bhidayasiri, D. Tarsy, Movement Disorders: A Video Atlas, Springer Science \& Business Media, 2012.

[10] E. Hametner, K. Seppi, W. Poewe, The clinical spectrum of levodopa-induced motor complications, Journal of Neurology. 257 (2010) 268-275.

[11] T. Shany, S.J. Redmond, M.R. Narayanan, N.H. Lovell, Sensors-based wearable systems for monitoring of human movement and falls, IEEE Sensors Journal. 12 (2012) 658-670.

[12] N.L.W. Keijsers, M.W.I.M. Horstink, S.C.A.M. Gielen, Automatic assessment of levodopainduced dyskinesias in daily life by neural networks, Movement Disorders. 18 (2003) 70-80. doi:10.1002/mds.10310.

[13] J.I. Hoff, E.A.H. Wagemans, J.J. Van Hilten, others, Accelerometric assessment of levodopainduced dyskinesias in Parkinson's disease, Movement Disorders. 16 (2001) 58-61.

[14] M.G. Tsipouras, A.T. Tzallas, G. Rigas, S. Tsouli, D.I. Fotiadis, S. Konitsiotis, An automated methodology for levodopa-induced dyskinesia: assessment based on gyroscope and accelerometer signals, Artificial Intelligence in Medicine. 55 (2012) 127-135. 
[15] A.J. Manson, P. Brown, J.D. O’sullivan, P. Asselman, D. Buckwell, A.J. Lees, An ambulatory dyskinesia monitor, Journal of. Neurolology, Neurosurgery and Psychiatry. 68 (2000) 196-201.

[16] A. Samà, C. Perez-Lopez, J. Romagosa, D. Rodriguez-Martin, A. Català, J. Cabestany, et al., Dyskinesia and motor state detection in Parkinson' s Disease patients with a single movement sensor, in: D. Haemmerich, D. Panescu, J. Pearce, P. Prakash, E. Sloane, P. Soda, et al., 34th Annual International Conference of the IEEE Engineering in Medicine and Biology Society, San Diego, 2012: pp. 1194-1197.

[17] S. Das, B. Amoedo, F. la Torre, J. Hodgins, Detecting Parkinsons's symptoms in uncontrolled home environments: A multiple instance learning approach, in: L. Faes, E. Ifeachor, C. James, J. Antonio Jo, Y. P. Kahya, P. Laguna, et al., 34th Annual International Conference of the IEEE Engineering in Medicine and Biology Society, SanDiego, 2012: pp. 3688-3691.

[18] M.I. Chelaru, C. Duval, M. Jog, Levodopa-induced dyskinesias detection based on the complexity of involuntary movements, Journal of Neuroscience Methods. 186 (2010) 81-89.

[19] M. Ghassemi, S. Lemieux, M. Jog, R. Edwards, C. Duval, Bradykinesia in patients with Parkinson's disease having levodopa-induced dyskinesias, Brain Research Bulletin. 69 (2006) 512-518.

[20] J. Gour, R. Edwards, S. Lemieux, M. Ghassemi, M. Jog, C. Duval, Movement patterns of peakdose levodopa-induced dyskinesias in patients with Parkinson's disease, Brain Research Bulletin. 74 (2007) 66-74.

[21] P.R. Burkhard, H. Shale, J.W. Langston, J.W. Tetrud, Quantification of dyskinesia in Parkinson's disease: validation of a novel instrumental method, Movement. Disorders 14 (1999) 754-763.

[22] A. Salarian, H. Russmann, C. Wider, P.R. Burkhard, F.J.G. Vingerhoets, K. Aminian, Quantification of tremor and bradykinesia in Parkinson's disease using a novel ambulatory monitoring system, IEEE Transactions on Biomedical Engineering. 54 (2007) 313-322. doi:10.1109/TBME.2006.886670.

[23] C.C. Yang, Y.L. Hsu, A review of accelerometry-based wearable motion detectors for physical activity monitoring, Sensors (Basel). 10 (2010) 7772-7788. doi:10.3390/s100807772.

[24] H. Gjoreski, M. Lustrek, M. Gams, Accelerometer Placement for Posture Recognition and Fall Detection, $7^{\text {th }}$ International Conference on Intelligent Environments, Nottingham, UK, 2011: pp. 47-54. doi:10.1109/IE.2011.11.

[25] M.J. Mathie, J. Basilakis, B.G. Celler, A system for monitoring posture and physical activity using accelerometers, 23rd Annual International Conference of the IEEE Engineering in Medicine and Biology Society, Istambul, Turkey, 2001: pp. 3654-3657. doi:10.1109/IEMBS.2001.1019627.

[26] a J. Hughes, S.E. Daniel, L. Kilford, a J. Lees, Accuracy of clinical diagnosis of idiopathic Parkinson's disease: a clinico-pathological study of 100 cases., Journal of Neurolology, Neurosurgery and Psychiatry. 55 (1992) 181-184. doi:10.1136/jnnp.55.3.181.

[27] A. Nieuwboer, W. de Weerdt, R. Dom, E. Lesaffre, A frequency and correlation analysis of motor deficits in Parkinson patients, Disability and Rehabilitation. 20 (1998) 142-150.

[28] A. Samà, C. Perez-Lopez, D. Rodriguez-Martin, J. Cabestany, J.M. Moreno, A. RodriguezMolinero, A Heterogeneous Database for Movement Knowledge Extraction in Parkinson 's Disease, European Symposium on Artificial Neural Networks, Computational Intelligence and Machine Learning. Bruges (Belgium), 24-26 April 2013: pp: 413-418. 
[29] D. Rodríguez-Martín, C. Pérez-López, A. Samà, J. Cabestany, A. Català, A Wearable Inertial Measurement Unit for Long-Term Monitoring in the Dependency Care Area, Sensors (Basel). 13 (2013) 14079-14104. doi:10.3390/s131014079.

[30] D. Rodriguez-Martin, A. Samà, C. Perez-Lopez, A. Català, J. Cabestany, A. Rodriguez-Molinero, SVM-based posture identification with a single waist-located triaxial accelerometer, Expert Systems with Applications. 40 (2013) 7203-7211. doi:10.1016/j.eswa.2013.07.028.

[31] G. Deuschl, P. Bain, M. Brin, Consensus statement of the Movement Disorder Society on Tremor. Ad Hoc Scientific Committee., Movement Disorders. 13 Suppl 3 (1998) 2-23. http://www.biomedsearch.com/nih/Consensus-statement-Movement-DisorderSociety/9827589.html.

[32] E.K. Antonsson, R.W. Mann, The frequency content of gait, Journal of Biomechanics. 18 (1985) 39-47. http://www.ncbi.nlm.nih.gov/pubmed/3980487.

[33] V.N. Vapnik, The Nature of Statistical Learning Theory, $2^{\text {nd }}$ Edition, Springer-Verlag, New York, 1995.

[34] S. Patel, K. Lorincz, R. Hughes, N. Huggins, J. Growdon, D. Standaert, et al., Monitoring Motor Fluctuations in Patients With Parkinson's Disease Using Wearable Sensors, IEEE Transactions on Information Technology in Biomedicine. 13 (2009) 864-873.

[35] S. Arlot, A. Celisse, others, A survey of cross-validation procedures for model selection, Statistics Surveys. 4 (2010) 40-79.

[36] D. Anguita, A. Ghio, L. Oneto, X. Parra, J.L. Reyes-Ortiz, Energy Efficient Smartphone-Based Activity Recognition using Fixed-Point Arithmetic, Journal of Universal Computer Science. 19 (2013) 1295-1314. 